\title{
Performance of self-compacting rubberized concrete
}

\section{Performance du béton autoplaçant à base de granulats en caoutchouc recyclés.}

\author{
Bensaci Hamza $^{1}$, Menadi Belkacem ${ }^{1}$, Kenai Said ${ }^{1}$ et Yahiaoui Walid ${ }^{1}$ \\ ${ }^{1}$ Laboratoire de Géo-matériaux, Département de Génie Civil, Université de Blida1, Algérie
}

\begin{abstract}
Used tyre rubber wastes present a serious environmental problem of pollution and storage. The recycling of this waste in the industry of construction could be an appropriate solution to produce an eco-concrete and could contribute to the improvement of some of its properties. This paper aims to study the possibility of using tyre rubber waste as fine aggregate replacement in self-compacting concrete (SCC). Fines rubber particles of 0-2 $\mathrm{mm}$ of waste tyres were added SCC mixtures as a partial substitution of the total volume of sand at different percentages $(5,10,15,20$ and $30 \%)$. The influence of fines rubber of used tyres on fresh and hardened properties of the SCC was investigated. The fresh properties of SCC were performed by using slump-flow, T50 flow time, L-box, V-funnel and segregation resistance tests. Characteristics of the hardened state were obtained by compressive strength and thermal conductivity. The experimental results showed that the inclusion of fines rubber in SCC decreases the workability, reduced its passing capacity and increases the possibility of blocking. A decrease in compressive strength is observed with the increase in rubber content. On the other hand, the incorporation of the rubber fines aggregates enhances in a remarkably way the thermal conductivity.
\end{abstract}

Résumé : Les pneus usagés présentent des problèmes de pollution de l'environnement et de stockage. Le recyclage de ces déchets dans l'industrie de construction-s'avère une solution adéquate pour la production d'un éco-béton et peut contribuer à l'amélioration de certaines de ses propriétés. Cet article vise à étudier la possibilité de la valorisation des déchets caoutchouteux des pneus usagés sous forme de granulats dans le béton autoplaçant (BAP). Des particules de caoutchouc de 0-2mm de pneus ont été ajoutées aux mélanges du BAP en substitution partiel du volume total de sable à différents pourcentages $(5,10,15,20$ et 30\%). L'influence du caoutchouc sur les propriétés du BAP, aussi bien à l'état frais qu'à l'état durci, a été analysée. Les propriétés à l'état frais ont été déterminées à l'aide de tests du diamètre d'étalement et du temps d'écoulement, de L-box, du temps d'écoulement à l'entonnoir en $\mathrm{V}$ et de la résistance à la ségrégation par tamisage. Les caractéristiques à l'état durci ont été obtenues par l'essai de la résistance à la compression et la conductivité thermique. Les résultats expérimentaux ont montré que la présence du caoutchouc dans le BAP diminue l'ouvrabilité, réduit sa capacité de passage et augmente la possibilité de blocage. Une chute de la résistance à la compression est observée avec l'augmentation de taux de caoutchouc. Par contre, l'incorporation des granulats de caoutchouc améliore d'une façon remarquable la conductivité thermique. 


\section{Introduction}

Les pneus usagés présentent des problèmes de pollution de l'environnement et de stockage. Plus de 275 millions de pneus usagés sont stockés dans les décharges à travers les Etats Unis [1,2]. En Algérie, le nombre des pneus usagés est estimé à environ 1.5 millions par an [3]. L'élimination de ce type de déchets pose actuellement un sérieux problème pour les pays autour du monde en raison de leur effet direct sur l'environnement et la santé humaine [1, 4]. Le recyclage de ces déchets dans l'industrie de construction s'avère une solution adéquate pour la production d'un éco-béton et peut contribuer à l'amélioration de certaines de ses propriétés. A cet effet ,'utilisation des particules de caoutchouc des pneus usagés comme des granulats dans le béton a reçu une attention particulière au cours des deux dernières décennies par plusieurs chercheurs en raison de l'accroissement énorme du gisement des pneus usagés.

La majorité des travaux de recherches ont montré que les déchets des pneus usagés (caoutchouc ou fibre) peuvent être utilisés dans la matrice cimentaire comme substitution aux agrégats pour l'amélioration de certaines caractéristiques des bétons.

khaloo et al. [5], ont examiné l'utilisation des granulats en caoutchouc pour fabriquer le béton et une chute de la masse volumique du béton avec l'augmentation du taux de substitution de caoutchouc a été observée. Cependant, Siddique et Naik[6] ont rapporté aussi une augmentation de la teneur en air occlus dans la matrice cimentaire contenant des particules de caoutchouc qui a une réduction de la masse volumique. En outre, Topçu et al. [7], ont étudié des BAP incorporant des agrégats en caoutchouc (G.C) par la substitution des agrégats naturels par des G.C en poids avec différents types d'agent de viscosité. Ils ont constaté queles propriétés rhéologiques des BAP étudiés sont acceptables, quel que soit le taux de substitution du caoutchouc. Güneyisi et al. [8] ont étudié les propriétés mécaniques des bétons de caoutchouc par substitution des granulats naturels par des granulats en caoutchouc (de 2,5 à 50 \%) avec et sans l'utilisation de la fumée de silice. Les résultats ont montré une perte d'ouvrabilité ainsi qu'une forte réduction en termes de résistance avec l'augmentation du taux en caoutchouc. D'autre part, les auteurs ont indiqué que pour une teneur de $25 \%$ de caoutchouc on peut produire un béton avec une résistance à la compression de 16 à $32 \mathrm{MPa}$. De plus Eldin et Senouci [9] ont étudié l'influence de l'ajout de caoutchouc comme agrégats par la substitution totale du gravier ou du sable sur la résistance à la compression du béton. Ils ont observé une chute de résistance de l'ordre de $85 \%$ et $65 \%$ lorsqu'on substitue totalement le gravier et le sable par du caoutchouc, respectivement. Yung et al. [10], ont étudié l'effet du taux et la finesse du granulat en caoutchouc substitué au sable naturel par volume sur les performances du BAP. Ils ont observé une chute de résistance à la compression avec l'augmentation du taux de caoutchouc à l'exception du BAP avec 5\% du caoutchouc qui présente une meilleure résistance par rapport au béton de référence. L'incorporation du caoutchouc dans le béton joue un rôle important dans l'amélioration de la capacité de déformation du béton [11]. Afin d'exploiter les gisements du déchet pneumatique et préserver l'environnement dans une vision de développement durable, des études et des recherches intensives et plus détaillé sont nécessaires. Le but de cet article est d'analyser l'effet des granulats de caoutchouc des pneus usagés sur les performances du béton autoplaçant à l'état frais et durci.

\section{Matériaux et essais}

Pour tous les mélanges de béton autoplaçant réalisés dans la présente étude, nous avons utilisé un ciment Portland CEM II / B 42.5Nde finesse $4640 \mathrm{~cm}^{2} / \mathrm{kg}$; deux types de granulats grossiers $3 / 8$ et $8 / 15$ d'origines calcaires concassés de densité $2.53 \mathrm{~kg} / \mathrm{m}^{3}$ et de $1 \%$ d'absorption d'eau et un sable fin de dune avec un module de finesse de 2,56, une densité de 2,61 et une absorption d'eau de $1,34 \%$. Les granulats en caoutchouc utilisés sont obtenus à partir du broyage de pneus usagés, leur densité est de 0,77 et un coefficient d'absorption d'eau égale 0,24 et leur taille maximale est de $2 \mathrm{~mm}$. Un plastifiant 
polycarboxylate (MEDAFLOW 30) de densité spécifique de $1,06 \mathrm{~kg} / \mathrm{m}^{3}$ est utilisé.

Dans la présente étude, la méthode japonaise est choisie pour la formulation des différents mélanges du béton autoplaçant étudiés. Notre formulation consiste à maintenir un rapport sable/mortier(S/M) de $50 \%$, un super-plastifiant (SP) de $1 \%$ en poids de ciment et un rapport $\mathrm{E} / \mathrm{L}=0.42$ constant dans tous les mélanges. Six mélanges ont été réalisés avec différents pourcentages de caoutchouc $(0 \%, 5 \%, 10 \%, 15 \%, 20 \%$ et $30 \%)$ comme substitution du sable en volume. Le détail de la composition des mélanges des différents BAP étudiés sont données dans le tableau 1.

Table 1. Compositions de mélanges des BAPs.

\begin{tabular}{lcccccc}
\hline Constituants & \multicolumn{6}{c}{$\left(\mathbf{k g} / \mathbf{m}^{\mathbf{3}}\right)$} \\
\cline { 2 - 7 } & Réf & $\mathbf{5 \%}$ & $\mathbf{1 0 \%}$ & $\mathbf{1 5 \%}$ & $\mathbf{2 0 \%}$ & $\mathbf{3 0 \%}$ \\
Ciment & & \multicolumn{6}{c}{442.4} \\
Gravier & & & 812.9 & & \\
Sable & 819.7 & 807 & 794.5 & 781.8 & 769.2 & 744 \\
Caoutchouc & - & 12.6 & 25.2 & 37.8 & 50.4 & 75.7 \\
SP & & & & 4.42 & & \\
Eau & & & & 200.7 & & \\
\end{tabular}

Pour évaluer les performances rhéologiques des mélanges des BAP, l'essai d'étalement, l'essai à l'entonnoir en V, l'essai L-box et la résistance à la ségrégation par tamisage ont été réalisés sur la base des recommandations d'EFNARC [12].Après la réalisation des essais à l'état frais des BAP, des éprouvettes cubiques de $100 \mathrm{~mm}$ de côté et prismatiques $\left(70 \times 70 \times 280 \mathrm{~mm}^{3}\right)$ ont été confectionnées pour l'essai de résistance à la compression et l'essai de retrait total, respectivement. Les éprouvettes cubiques destinées à l'essai de compression ont été conservées dans l'eau à une température de $20 \pm$ $2^{\circ} \mathrm{C}$ pendant 28 jours avant l'essai. Par contre, les éprouvettes prismatiques ont été soumis directement à l'essai du retrait total après démoulage à une humidité de $60 \%$ et une température $20 \pm 2{ }^{\circ} \mathrm{C}$ jusqu'à l'âge 28 jours. Pour déterminer les valeurs des coefficients de conductivité thermique des BAPà 28 jours, on a utilisé le dispositif du fil chaud de CT-mètre conforme à la norme NF EN 993-15 sur des éprouvettes cubiques10x10x10 cm.

\section{Résultats et discussion}

\subsection{Propriétés du BAP à l'état frais}

Les résultats de l'étalement de tous les mélanges des bétons autoplaçants contenant des granulats caoutchouteux comme substitution partielle du sable sont illustrés sur la figure 1.En comparant, les résultats des différents étalements avec les recommandations du béton autoplaçant[12], on constate que tous les mélanges du BAP présentent un étalement acceptable à l'exception du BAP avec 30\% GC.D' après cette figure, on remarque que le diamètre d'étalement diminue avec l'augmentation du taux d'incorporation des granulats en caoutchouc. Cette diminution est de l'ordre de $1,9 \%, 5,3 \%, 8,6 \%, 11,2 \%$ et $31.6 \%$ pour des taux de substitution de $5 \%, 10 \%, 15 \%$, $20 \%$ et $30 \%$ GC, respectivement en comparaison avec le $\mathrm{BAP}$ de référence. Cette réduction peut être attribuée à la

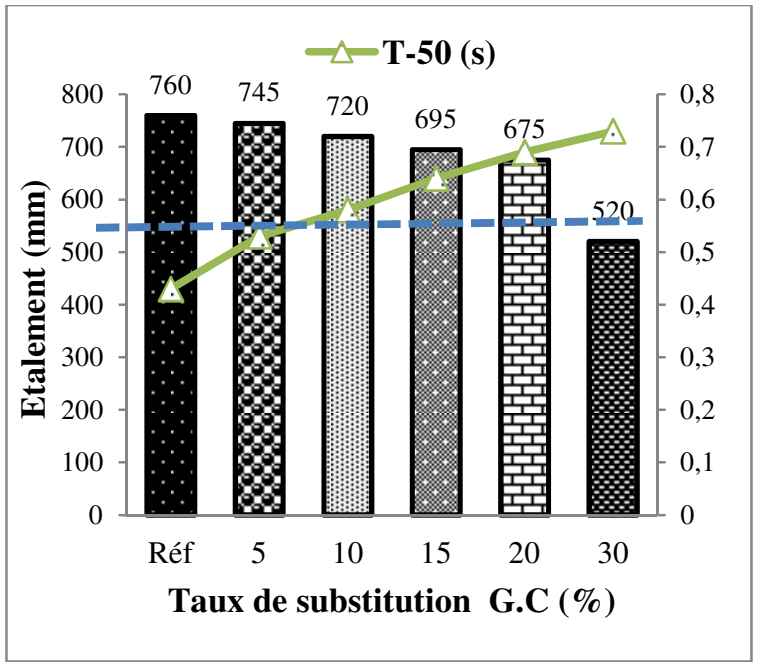

Fig. 1. Effet de l'incorporation du G.C sur l'étalement et le T50.

rugosité de la surface et la forme sphérique des particules de caoutchouc [11].Le temps d'étalement, T50 est inversement proportionnel à l'étalement comme l'indique la figure 1. Les mélanges du BAP avec $20 \%$ et $30 \%$ de granulats caoutchouteux donnentdes valeurs plus élevées du T50, ce qui indique une viscosité élevée en comparaison avec le BAP de référence. La même 
tendance est observée pour le temps d'écoulement du Vfunnel (Fig. 2). Comme l'indique la figure 2, pour tous les mélanges étudiés le temps d'écoulement est entre 2.2 et 6.4 secondes. Ceci montre que les mélanges du BAP avec et sans granulats en caoutchouc étudiés sont conformes aux recommandations d'EFNAC. Le comportement du béton incorporant des G.C dans une zone ferraillée qui est traduit par l'essai de la boite en L-box est étudié. Cet essai donne une bonne évaluation de la capacité de passage et de remplissage.

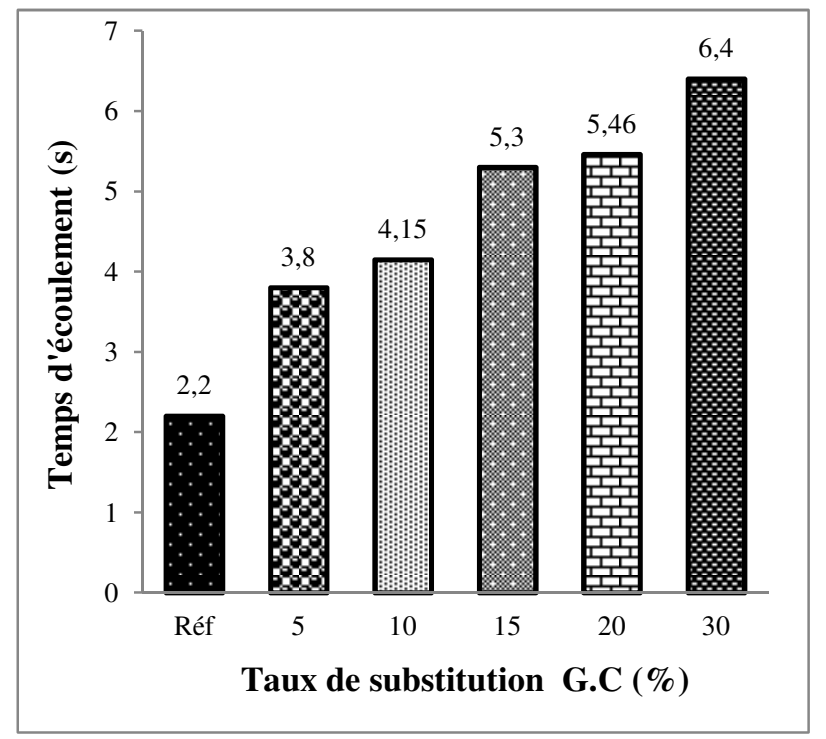

Fig. 2. Effet de l'incorporation du G.C sur le temps d'écoulement en V-funnel.

Les résultats de l'essai L-box exprimés par le rapport $\mathrm{H} 2 / \mathrm{H} 1$ de tous les mélanges étudiés dans la présente étude sont illustrés sur la figure 3. Les valeurs mesurées de la capacité de passage $(\mathrm{H} 2 / \mathrm{H} 1)$ varient de 0.98 à 0.53 . D'après la figure 3 , on remarque que les mélanges avec 15, 20 et $30 \%$ de GC présentent un blocage au niveau des armatures du L-box et ne satisfait pas les exigences recommander pour les BAPs. Les mélanges du BAP avec des taux de substitution du GC supérieurs à $15 \%$ présentent une viscosité élevée ce qui empêche le passage à travers les trois barres du dispositif L-box. Par contre, les autres mélanges du BAP présentent des rapports $\mathrm{H} 2 / \mathrm{H} 1$ admissibles.

Les résultats de l'effet de la variation du taux d'incorporation des G.C sur la résistance à la ségrégation des BAP sont donnés sur la figure 4. Selon cette figure, on remarque que l'augmentation du taux d'incorporation des G.C s'est traduite par une diminution du taux de ségrégation. Pendant l'essai, aucun signe de ségrégation n'a été détecté pour tous les mélanges des bétons autoplaçants.

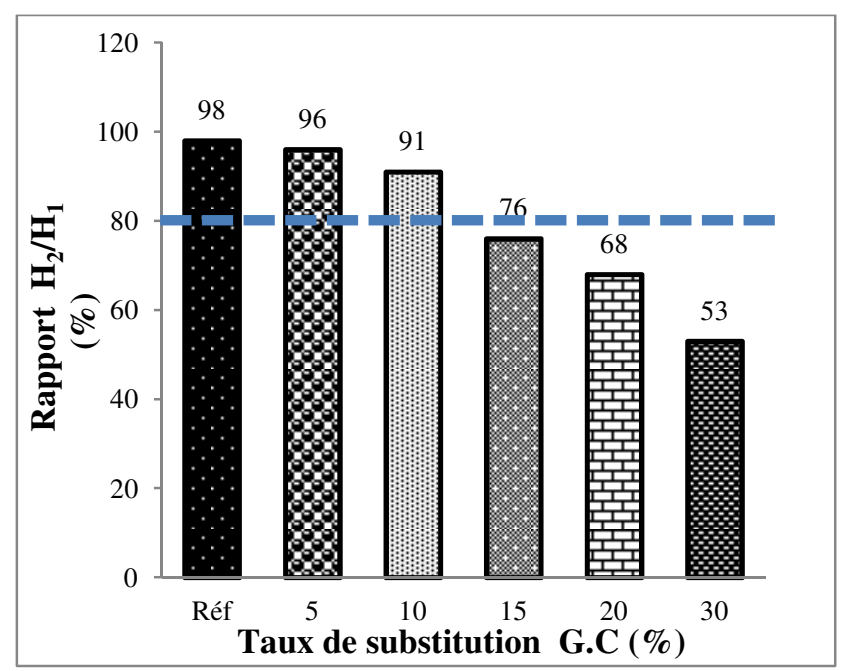

Fig. 3. Effet de l'incorporation du G.C sur le rapport H2/H1.

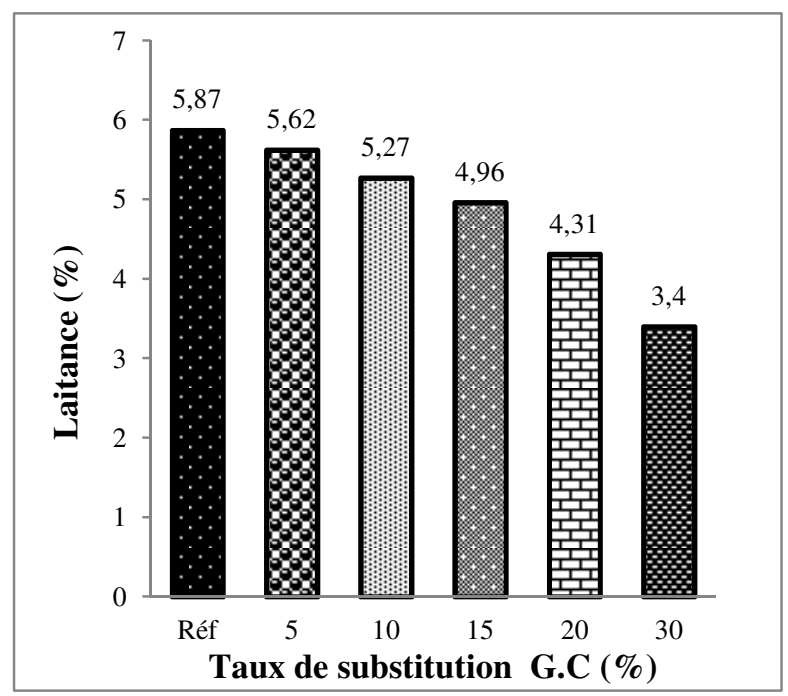

Fig. 4. Résistance à la ségrégation en fonction taux de substitution en G.C.

Ces résultats sont contradictoires à ceux obtenus par Garros [11] et Cuong [13] qui ont trouvé que l'utilisation des gros G.C de classe $(0 / 4 \mathrm{~mm})$, entraine un phénomène de ségrégation contrairement à notre cas d'étude ou nous avons utilisé des G.C de classe $(0 / 2 \mathrm{~mm})$.

\subsection{Effet de l'incorporation des G.C à l'état durci}

\subsubsection{Résistance à la compression}


Les résultats indiquent clairement (Fig.5) que l'incorporation de G.C est très préjudiciable vis à vis de la résistance à la compression. A titre d'exemple, un taux de substitution en G.C. de 15 \% entraîne une chute de résistance remarquable de 45,4\% par rapport au béton de référence. Ceci peut être expliqué par la faible rigidité du caoutchouc par rapport à celle des granulats naturels selon $\mathrm{Li}$ et al. [14]. La deuxième raison est le défaut d'adhérence entre le caoutchouc et la matrice cimentaire et la mauvaise qualité de la zone de contact entre ces deux phases. Cet argument a été aussi avancé par Garros [11] qui montre que la zone de transition entre la matrice et le granulat en caoutchouc est peu compacte et présente une porosité importante.

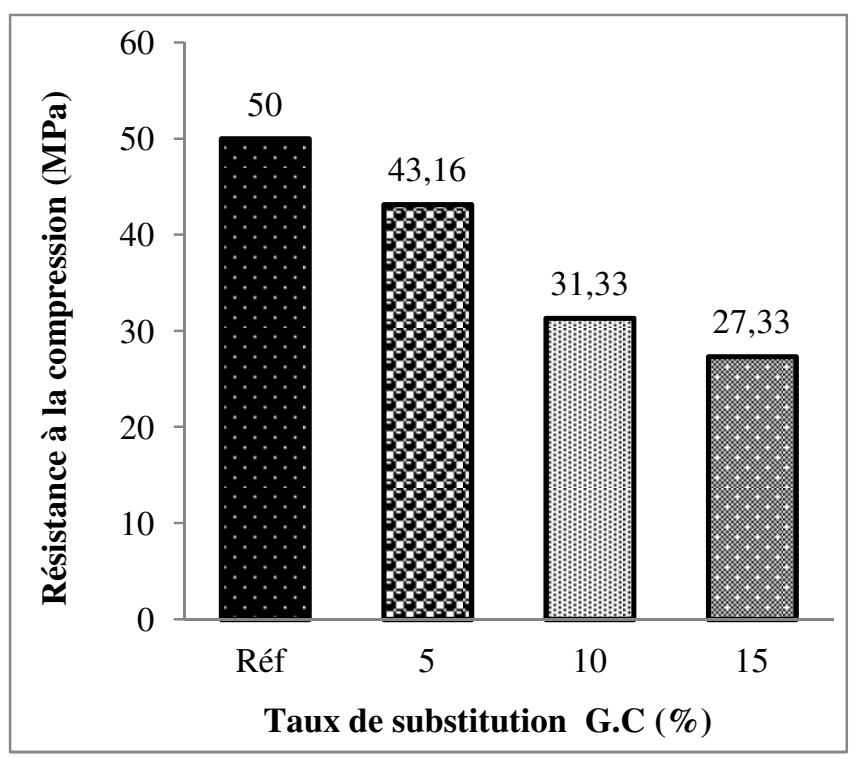

Fig. 5. Effet des G.C. sur la résistance à la compression à 28 jours.

\subsubsection{Conductivité thermique}

Les résultats des mesures de la conductivité thermique à l'état durci des bétons sont donnés sur la figure 6. Les résultats ci-dessus montrent que la conductivité thermique diminue avec l'augmentation du dosage des granulats caoutchouc. Par exemple, la présence de G.C. entraîne une réduction de la conductivité thermique de $26.50 \%$ lorsqu'on augmente les G.C de 0 à 15\%. Cette amélioration peut s'expliquer par la porosité élevée du BAP avec l'adjonction de G.C. D'après Benazzouk [15] l'air entraîné lors du malaxage conduit à une porosité fermée dans la matrice cimentaire, ce qui améliore encore les performances d'isolation du composite cimentaire incorporant des G.C.

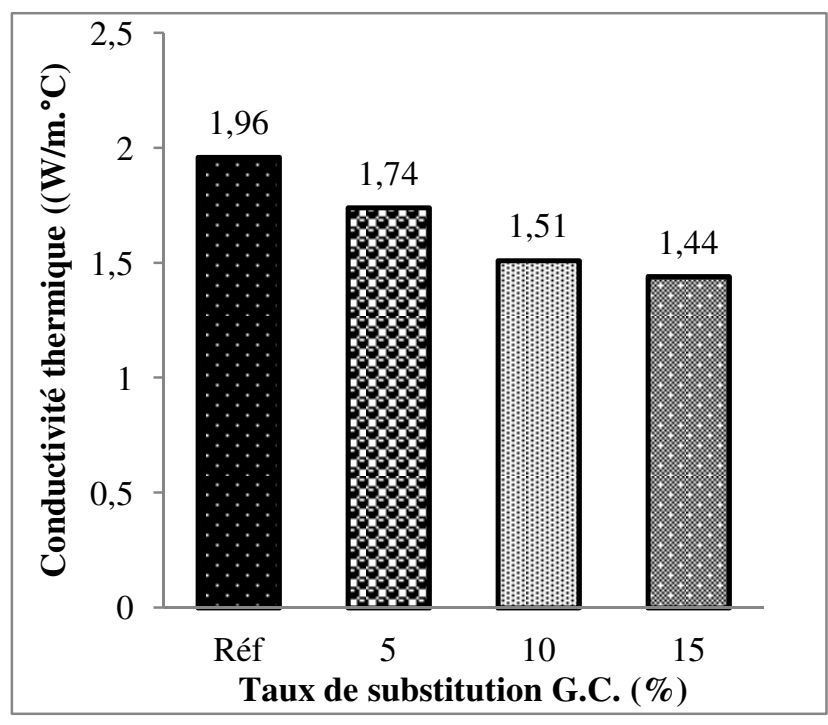

Fig. 6. Influence du dosage en G.C. sur la conductivité thermique.

\section{Conclusion}

Les résultats obtenus de la présente étude peuvent être résumés comme suit :

-L'incorporation des granulats en caoutchouc diminue les propriétés rhéologiques et conduit à des mélanges des BAP beaucoup plus visqueux à cause de la surface rugueuse de ces granulats.

- L'incorporation des granulats en caoutchouc est très préjudiciable vis-à-vis de la résistance à la compression.

-La conductivité thermique diminue lorsque le taux d'incorporation en G.C. augmente.

-Des bétons autoplaçants conformes aux normes peuvent être fabriqués avec l'utilisation d'un taux de remplacement du caoutchouc $(0-2 \mathrm{~mm})$ par du sable allant jusqu'à $15 \%$.

\section{References}

[1] A.Meddah, M. Beddar, A. Bali,use of shredded rubber tire aggregates for roller compacted concrete Pavement,Journal of Cleaner Production, pp. 187-192, (2014).

[2] M. R. Hall, K. B.Najim, Structural behaviour and 
durability of steel-reinforced structural Plain/SelfCompacting Rubberised Concrete (PRC/SCRC), Construction and Building Materials, no. 73 , p. 490-497, (2014).

[3] H. Trouzine, A. Asroun, N. Asroun, F. Belabdelouhab, N. T. Long, Problématique des pneumatiques usagés en Algérie Nature \& Technologie, no. 05, (2011).

[4] M. Gesoglu, E. Guneyisi, Permeability properties of self-compacting rubberized concretes, Construction and Building Materials, no. 25, p. 3319-3326, (2011).

[5] A. R. Khaloo, M. Dehestani, P. Rahmatabadi, J.Mechanical properties of concrete containing a high volume of tire-rubber particles, WasteManag. 28, 24722482, (2008).

[6] Siddique, R., Naik, T.R,Properties of concrete containing scrap-tire rubber: an overview,Waste Manag. 563-569, 2004.

[7] I. B. Topcu, T. Bilir, Experimental investigation of some fresh and hardened properties of rubberized selfcompacting concrete, Materials and Design, vol. 30, no. 123, p. 3056-3065, (2009).

[8] E. Güneyisi, M. Gesoğlu,T. Özturan, Properties of rubberized concretes containing silica fume,Cement and Concrete Research 34, 2309-2317, (2004).

[8] E. Güneyisi, M. Gesoğlu, T. Özturan, Cement and Concrete Research 34, 2309-2317, (2004).

[9] N. Eldin, A.B Senouci, Rubber-Tire Particles asConcrete Aggregate,J. Mater. Civ. Eng, 478-496. (1993).

[10] W. H. Yung,L. C. Yung, L. H. Hua, A study of the durability properties of waste tire rubber appliedto selfcompacting concrete,vol. 41, no.122, p. 665-672, (2013).

[11] M .Garros, Composites cimentaires incorporant des granulats caoutchouc issus du broyage de pneus usagés : optimisation de la composition et caractérisation, Thèse de doctorat, Université Paul Sabatier de Toulouse, (2007). [12] EFNARC, Specifications and Guidelines for SelfCompacting Concrete English ed., European Federation for Spec Constr Chem and Concr Syst., (2005).

[13] H. A. Cuong. Optimisation de la composition et caractérisation d'un béton incorporant des granulats issus du broyage de pneus usagés : Application aux éléments de grande surface (Thèse de doctorat), INSA de Toulouse,

France,(2010).http://www.theses.fr/2010ISAT0024.

[14] Z. Li, F. Li, Li. J. S. L, Properties of concrete incorporating rubber tyre particles, Magazine of Concrete Research, 50, No4, 297-304, (1998).

[15] Benazzouk. A., Contribution à la valorisation de déchets de caoutchouc: composites cimentaires à base de caoutchouc compact et cellulaire. (Thèse de doctorat), université d'Amines, France, (2002). 